\title{
423 - Characteristics of specialized units for people with dementia and very severe challenging behavior in the Netherlands: a mixed method study
}

Gerrie Van Voorden, Mijke Lips, Sytse Zuidema, MD, Richard Christiaan Oude Voshaar, PhD, Martin

Smalbrugge, MD, PhD, Anne M.A. Van Den Brink, Anke Persoon, PhD RN, Raymond T. Koopmans, MD, PhD, Debby L. Gerritsen, PhD

Introduction: Little is known about the raising number of specialized units for patients with dementia and very severe challenging behavior in the Netherlands. This study describes organizational and treatment characteristics of a sample of these units.

Methods: The organizational and treatment characteristics were studied with digital questionnaires completed by the unit managers, interviews with the main physician(s) and observation of the physical environment. The questionnaire consisted of questions about general patient characteristics, unit characteristics and staff characteristics. Furthermore, an interview was held with the main/treating physician often together with another physician or psychologist. The interview guide consisted of questions about admission criteria, the role of staff involved and the treatment process.

Results: Thirteen units participated. Five units were part of a mental health $(\mathrm{MH})$ institution, seven units were part of a nursing home $(\mathrm{NH})$ organization and one unit was a cooperation of $\mathrm{MH}$ and $\mathrm{NH}$. Unit sizes ranged from 10 to 28 places. Ten of thirteen units started in 2010 or later. The age of patients admitted was estimated at 75 years. The percentage of involuntary admitted patients was $53 \%$ at $\mathrm{MH}$-units and $18 \%$ at $\mathrm{NH}$-units. Unit managers mentioned that due to a difference in reimbursement between $\mathrm{MH}$ and $\mathrm{NH}$ units had difficulty providing the specialized care. Another problem managers faced was recruiting nursing staff. Units strived for expertise in general staffing from both $\mathrm{MH}$ and $\mathrm{NH}$. The education level of the nursing staff was comparable between $\mathrm{MH}$ and $\mathrm{NH}$. At every unit a physician with background in elderly care medicine or geriatrics and a psychiatrist was involved. Interviewees stressed the role of the nursing staff in the treatment. They were key in providing the care and treatment that, since the main goal of interventions is treatment of and coping with challenging behavior.

Conclusion: The main finding of this study is that units caring for patients with dementia and challenging behavior, despite barriers in regulations and staffing shortage, search for combining expertise from nursing home care and psychiatry in their treatment.

\section{4 - Cardiac Healthcare Disparities in Schizophrenia at the End-of-Life}

Authors: Baruth, J., Bateman, D., Bateman, P. Kovacs, R., Lapid, M.

Background: Schizophrenia is a serious mental illness associated an early mortality of 15 to 20 years. Eighty percent of deaths are due to cardiovascular disease, and the risk of sudden cardiac death is threetimes greater than the general population. Both modifiable and non-modifiable risk factors like lifestyle, medication side-effects, genetics, and healthcare disparities have been identified, but this relationship is not fully understood.

Research Objective: To examine cardiac-related healthcare utilization of individuals with schizophrenia at the end-of-life.

Method: As a retrospective cohort study the Mayo Clinic Unified Data Platform (UDP) was used to identify a schizophrenia group (SG) $(n=610) 50$ years or older with a death date between $1 / 1 / 1999$ $1 / 1 / 2019$ and control group $(n=610)$ matched by gender ( $53 \%$ women) and age of death $(72.8 \pm 12.4$ years). Measures of cardiovascular healthcare utilization were evaluated within a 12-month period prior 
to death. Pearson's chi-square ( $\chi 2$ ), analysis of variance was used (ANOVA), and logistic regression were used for statistical analysis.

Results: SG was more likely than controls to be unmarried, unemployed, or from racial minority groups (all $p<0.001$ ) and was more likely to have diabetes mellitus $(p<0.001)$ or cardiovascular disease $(p=0.004)$. SG was less likely to receive an electrocardiogram (ECG) $(p<0.001)$, echocardiogram $(p=0.003)$, or cardiac catheterization procedure $(p<0.001)$, and more likely to receive hemoglobin A1C testing $(p<0.001)$. Of those receiving an ECG, SG had a greater mean QTc interval (453.8 ms vs. 438.0 ms; $p<0.001)$ and were twice as likely to have an ECG result interpreted as "prolonged QTc" $(p<0.001)$. Between group differences for utilization of troponin or low-density lipoprotein testing, or pacemakerrelated procedures were not statistically significant.

Conclusion: Individuals with schizophrenia in this cohort were less likely to receive cardiovascular evaluations and interventions during their last year of life. Despite the higher likelihood for prolonged QTc, a recognized biomarker of cardiac risk, SG was less likely to receive an ECG. Given the greater cardiac comorbidity and higher risk of sudden cardiac death in schizophrenia, interventions are needed to address these disparities in care.

\section{5 - Incorporating young children into family caregiving for people with dementia in Taiwan.} Author List: Ching-Yi Kuo, MA. Shu-Chuan Chen, PhD. National Tainan Junior College of Nursing.

\section{Background:}

Positive attitudes and appropriate knowledge about dementia are essential for the provision of suitable dementia care. Children as future voters may take on a critical role to increase community awareness and knowledge about dementia. Guided by Chinese filial piety cultural virtues, Taiwanese children are taught to respect and care for family seniors and extend this respect to other seniors. Accordingly, young children are considered part of family dementia caregiving system, despite children rarely assume formal caregiver role and not directly provide care work. However, Taiwanese children may possess stigmatized attitudes toward dementia due to a lack of proper dementia knowledge.

\section{Research Objective:}

This study aims to survey Taiwanese children age 9-11 years old attitudes and knowledge to the concepts of dementia.

Methods: A total of 312 Taiwanese children from 8 elementary schools in the south Taiwan, aged 9-11 years old, were surveyed to assess their knowledge and attitudes toward dementia. Participants completed The Kids Insight into Dementia (KIDS), which is a 5-scale questionnaire containing three factors, "Personhood", "Stigma", and "Dementia Understanding". The data was analyzed using quantitative method.

Results: The results show that Taiwanese children are unfamiliar with dementia as related to brain disease $(M=3.94, S D=2.91)$, Taiwanese children are unfamiliar about nursing home provision of care $(M=3.97, S D=2.17)$, Taiwanese children do not know the course of dementia disease ( $M=3.34, S D-1.39)$. Participants scored high on the question of "people with dementia have hobbies and interests" ( $M=4.15$, $\mathrm{SD}=1.22)$; Participants reported that they agreed less often with stigma attached to the people with dementia, including items of "I would feel a bit scared if I met someone dementia in the street" $(M=2.70, S D=1.79)$, "people with dementia can be creepy" ( $M=2.18, S D=1.71)$, "It would be annoying of frustrating to spend time with someone with dementia" ( $M=2.15, S D=1.17)$, "It is unlikely that I would meet someone with dementia" $(M=2.36, S D=1.27)$. 